\title{
Gaia: how to map a billion stars with a billion pixels
}

\author{
J. H. J. de Bruijne ${ }^{1}$ \\ ${ }^{1}$ ESA/ESTEC/RSSD/SCI-SA (Astrophysics \& Fundamental Physics Missions Division), \\ Postbus 299, 2200 AG Noordwijk, The Netherlands \\ Email: jdbruijn@rssd.esa.int
}

\begin{abstract}
Gaia, ESA's ambitious star-mapper mission due for launch late-2011, will provide multi-epoch micro-arcsecond astrometric and milli-magnitude photometric data for the brightest one billion objects in the sky, down to at least magnitude 20. Spectroscopic data will simultaneously be collected for the subset of the brightest 100 million stars, down to about magnitude 17. This massive data volume will allow astronomers to reconstruct the structure, evolution and formation history of the Milky Way. It will also revolutionize studies of the solar system and stellar physics and will contribute to diverse research areas, ranging from extra-solar planets to general relativity.

Underlying Gaia's scientific harvest will lie in a Catalogue, built on the fundamental spacebased measurements. During the 5-year nominal operational lifetime, Gaia's payload, with its CCD mosaic containing 1 billion pixels, will autonomously detect all objects of interest and observe them throughout their passage of the focal plane. This paper discusses the workings of the Gaia instrument, details its payload, and discusses in depth how the scientific measurements will be collected. It addresses issues like maintenance of the scanning law, on-board data processing, the detection and confirmation of objects (single and multiple stars), the detection and rejection of cosmic rays and solar protons, the fundamental science measurements themselves composed of windows of CCD samples (pixels), and special strategies employed to maximize the science return for moving (i.e., solar-system) objects. The paper also explains how an on-board priority scheme will ensure catalogue completeness down to the faintest magnitudes possible, despite the limited ground-station availability and the enormous data volume that will be sent to the ground.
\end{abstract}

Keywords. space vehicles: instruments, surveys, astrometry, instrumentation: detectors, instrumentation: photometers, instrumentation: spectrographs

\section{Introduction and conclusion}

Gaia has received ample attention at this conference. Invited talks on Gaia's science and the Gaia Data Processing and Analysis Consortium (DPAC) have been delivered by L. Lindegren and F. Mignard (see Lindegren 2008 and Mignard 2008). Given the limited space allocated to this paper in these proceedings, we have decided to present our poster in Figure 1 and to refer the interested reader to the above-mentioned contributions in these proceedings and, of course, to the Gaia website at http://www.rssd.esa.int/Gaia for more details about the Gaia mission.

\section{Acknowledgements}

We would like to thank the symposium organizers for a very pleasant and instructive meeting. We would like to acknowledge the useful comments we received on the poster from K. O'Flaherty, T. Prusti, and A. Short. The poster layout was kindly provided by 


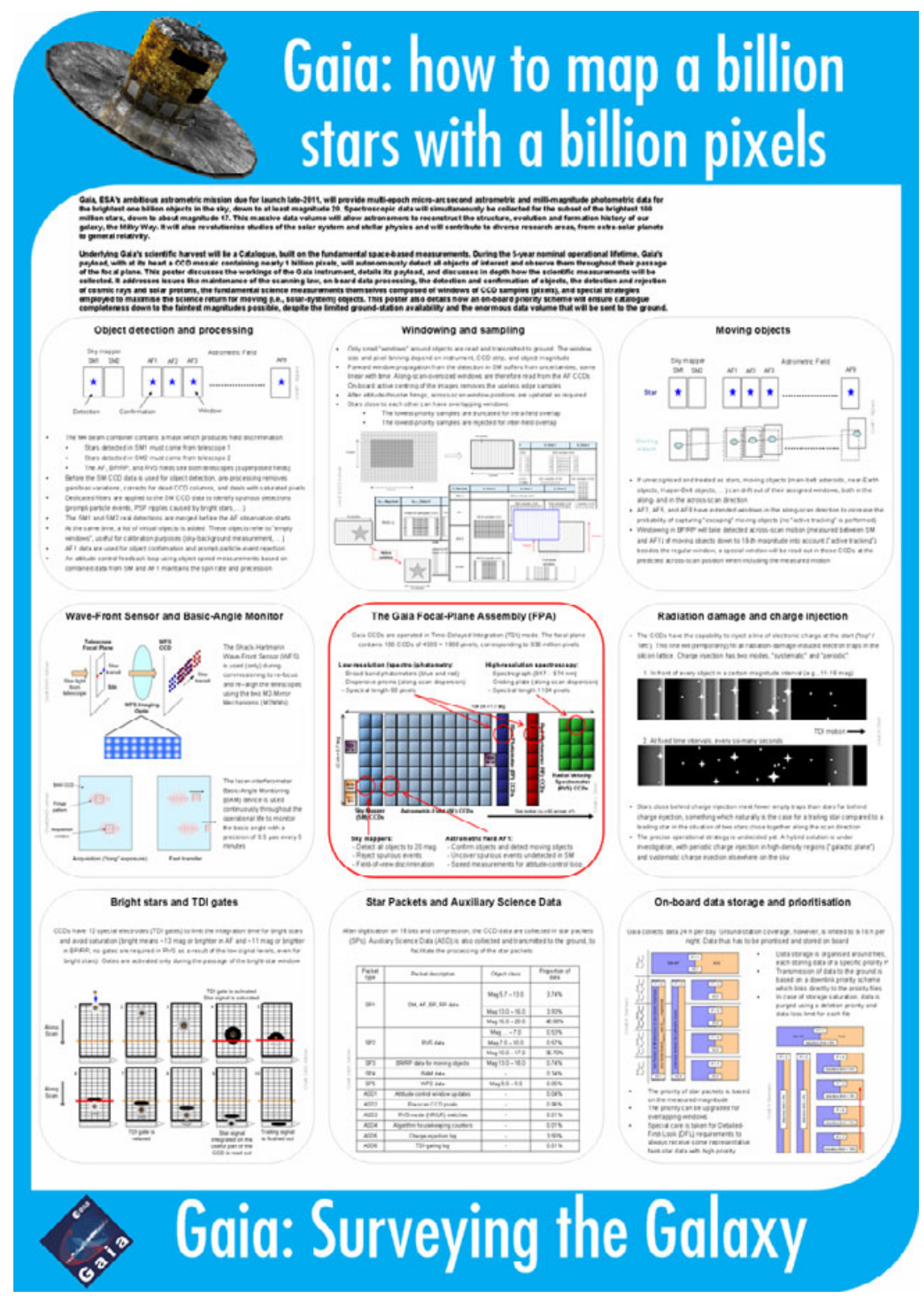

Figure 1. Poster presented at IAU Symposium 248 by the author.

J. Douglas. The images used in the poster are courtesy of EADS Astrium, M. Biermann, A. Short, and F. Mignard.

\section{References}

Lindegren, A. 2008, in: Wenjing Jin, et al. (eds), A Giant Step: from Milli- to Micro-arcsecond Astrometry, Proc. IAU Symposium No. 248 (Cambridge University Press), this volume p. 217

Mignard, F. 2008, in: Wenjing Jin, et al. (eds), A Giant Step: from Milli- to Micro-arcsecond Astrometry, Proc. IAU Symposium No. 248 (Cambridge University Press), this volume p. 224 